\title{
THE PNEUMOCOCCIDAL POWER OF WHOLE BLOOD
}

\section{Description of Method. Results in Individuals with No History of Lobar Pneumonia ${ }^{1}$}

By W. D. SUTLIFF AND D. R. RHOADES

(From the Secand (Cornell) Medical Division and the Pathological Department of Bellevue Hospital, New York and the Hygienic Laboratory of the United States Public

Health Service)

(Received for publication May 27, 1929)

The immunological properties of whole blood in relation to the pneumococcus have been studied by various methods. Wright, A. E. (1) and his followers used the slide cell technique devised by him and based upon the use of defibrinated blood. Heist and his co-workers $(2,3)$ have also used defibrinated blood. Bull and Tao (4) have used blood protected against coagulation by the addition of sodium citrate. Various workers have studied the course of pneumococcus infection in the blood stream of experimental animals: Wright, H. D. (5), Goodner, K. $(6,7)$, and Cecil and Blake $(8,9,10,11,12,13)$. The most recent work on phagocytic immunity is that of Robertson and his coworkers $(14,15,16)$ with serum-leucocyte mixtures. The methods described below are in part adaptations of those used by these lastnamed investigators. In the early part of this study serum-leucocyte mixtures were tried repeatedly, but the manipulations required to free the leucocytes from the whole blood were, in our experience, too numerous and delicate for practical use. It occurred to us that whole uncoagulated blood might possibly present similar immunological features and that if this were so, it could be substituted with advantage for the serum-leucocyte mixtures.

\section{MATERIALS AND METHODS}

An agitator was constructed as illustrated in figure 1. The motive power of the agitator is a 4 -volt storage battery. Speed is regulated by a rheostat starting key

${ }^{1}$ This work also received financial aid from the Research Fund of the Metropolitan Life Insurance Company. 
at $R$. The eccentric wheel, on the circumference of which the tubes are placed, provides rotation and end to end mixing of the contents of the tubes.

Heparin (Howell, 17,18), was used as the anticoagulant. Mechanical defibrination changes the white blood cells by destroying or removing many of the polynuclear cells as shown by Bull (20). Potassium or lithium oxalate in concentration necessary for preventing coagulation has some bacteriostatic action in cultures of pneumococci. Heparin in the concentration used in the blood in this study has no apparent effect on the growth of the pneumococcus.

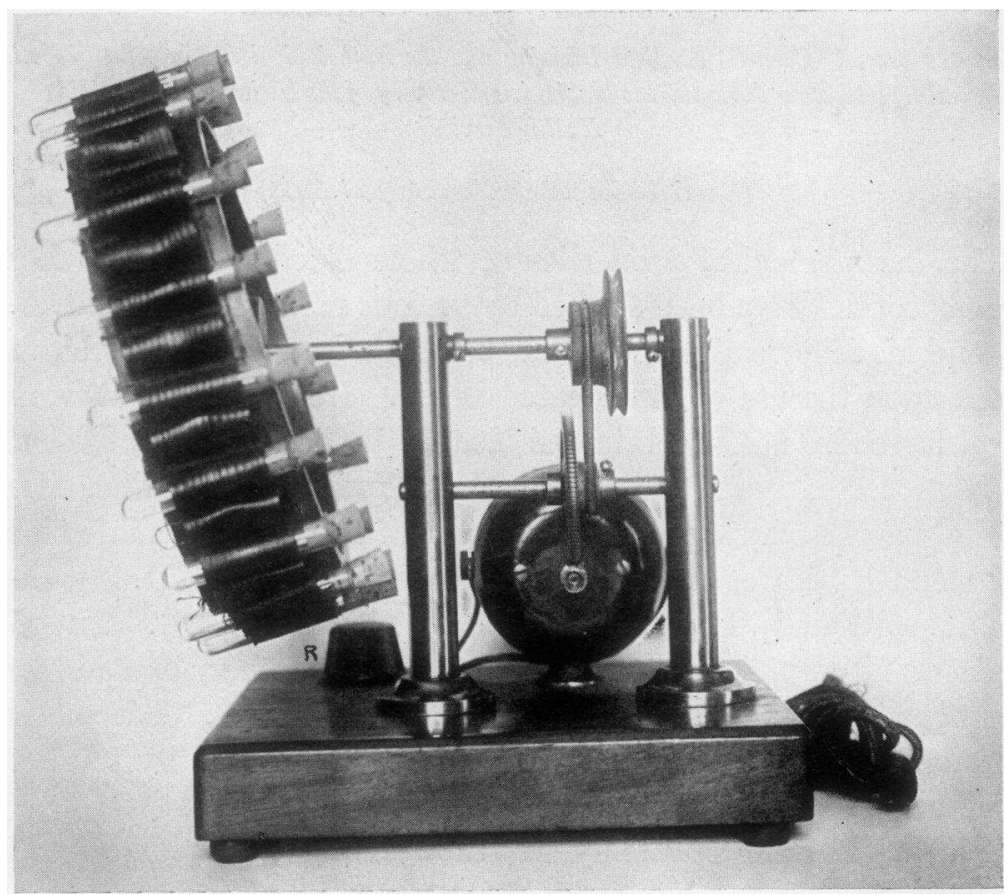

Fig. 1. Eccentric Rotator to Mix Tube Contents

Since the completion of this work the anti-complementary action of heparin has been studied by Ecker and Gross (19). We have been able to confirm this action of heparin when added directly to serum and also when used as an anticoagulant in whole blood. The amounts of heparin needed to demonstrate anti-complementary power are, however, greater than any that were used in this study. We feel it safe to say that in the whole blood samples used in this study, the plasma would in all probability have been active in the usual sheep cell and antigen system.

The test was performed as follows:

The blood sample to be tested was collected under sterile precautions, in 5 to 
$15 \mathrm{cc}$. amounts in a pyrex flask containing $1 \mathrm{mgm}$. of heparin in $0.1 \mathrm{cc}$. of normal saline for each $5 \mathrm{cc}$. of blood. The flask was kept in a dish of cracked ice before receiving the blood and from 5 to 10 minutes thereafter. A total white cell count and a differential count were made on the blood in the flask after it was received in the laboratory.

Three cubic centimeters of an 18 hour broth culture of Type I pneumococci of a virulence sufficient to kill 20 gram mice regularly in a dilution of $10^{-8}$ were seeded in $25 \mathrm{cc}$. of sterile broth in a $50 \mathrm{cc}$. centrifuge tube. This culture became dense enough in 3 to 4 hours to appear opaque at the greatest diameter of the tube (11 inches). It was centrifugalized at high speed for 15 minutes, the supernatant broth poured off, and the organisms taken up in $5 \mathrm{cc}$. of gelatin-Locke's solution. The density of this suspension was read by the Gates method (21) and diluted

TABLE 1

Contents of tubes when assembled

\begin{tabular}{c|c|c|l}
\hline Tube number & Blood & Pneumococci & \multicolumn{1}{|c}{ Obtained from 0.1 $\propto$. of } \\
\cline { 2 - 3 } 1 & $c c$. & & \\
2 & 0.5 & $10^{8}$ & Standard suspension \\
3 & 0.5 & $10^{7}$ & Dilution 1 \\
4 & 0.5 & $10^{\circ}$ & Dilution 2 \\
5 & 0.5 & $10^{5}$ & Dilution 3 \\
6 & 0.5 & $10^{4}$ & Dilution 4 \\
7 & 0.5 & 1,000 & Dilution 5 \\
8 & 0.5 & 100 & Dilution 6 \\
9 & 0.5 & 100 & Dilution 6 \\
& 0.5 & 0 & Gelatin-Locke's solution \\
\hline
\end{tabular}

to a standard density equivalent to approximately 1000 million pairs of pneumococci per cubic centimeter. The reading for this density with the Gates nephelometer was $1.1 \mathrm{~cm}$. for the work to be reported.

Dilutions of the standard suspension of pneumococci were made in gelatinLocke's solution. Each dilution was 1:10 of the preceding dilution.

The culture dilutions were now placed in the 9 small tubes, as indicated in table 1. A $1 \mathrm{cc}$. pipette with tip about $8 \mathrm{~cm}$. long was used to place the culture well in the bottoms of the tubes. One tenth cubic centimeter of dilutions 4, 5, 6, and 7 were plated in blood agar as a check on the number and viability of the pneumococci actually placed in a test.

The blood was then added. It was mixed by gentle rotation of the flask. Five tenth cubic centimeter amounts were introduced to the bottom of all the tubes, except the 8 th, with a $2 \mathrm{cc}$. pipette drawn out at the tip. The 8 th tube received $0.5 \mathrm{cc}$. of plasma obtained from the heparinized blood by centrifugalization.

The contents of the 9 tubes, when the test was completely assembled, are illustrated in table 1. 
The corks were then dipped in melted paraffin, cooled and placed in the tubes, after flaming the lips of the tubes slightly.

The tubes were inserted in the leather band on the eccentric wheel of the agitator and the time noted. The rheostat was adjusted so that the wheel revolved at the rate of 15 to 25 revolutions per minute. Agitation was continued for 6 hours; then the tubes were set upright in a rack and replaced in the incubator for 18 hours.

In reading the results of the test, the color change was recorded, the appearance of the growth in the plasma control tube noted as to density and uniformity, and pour-plate cultures were made from each of the tubes in the test. It has been our experience from repeated estimations and dilutions of such cultures that a plate that appears uniformly brown, indicated in protocols by the symbol, " $\alpha$," contains more than 5000 colonies. Any less than 5000 pneumococci can be estimated approximately and this estimation has been indicated by the symbol " $\alpha$-", meaning a lesser degree of growth than a "brown" plate, but still too great a number of colonies to be counted.

We have used the standard procedure for the mouse protection test as described in a number of text books.

\section{Consistency of the measurements}

Various checks were employed for testing the accuracy of the methods used for counting and distributing the pneumococci and white cells in the tubes. The system of standardizing and diluting the suspension of organisms was analyzed by making pour-plate cultures in blood agar with every performance of the test. Granting that the standardized suspension contained 1000 million pairs of pneumococci per cubic centimeter, and that the dilution of each succeeding tube by one tenth was perfectly carried out, 100 colonies should result from planting $0.1 \mathrm{cc}$. of the 6 th dilution. Dilutions 5 and 7 should contain 1000 and 10 organisms, respectively, in each $0.1 \mathrm{cc}$. As a matter of record, the variation in the number of colonies obtained by planting $0.1 \mathrm{cc}$. of the 6th dilution in 15 consecutive tests was from 35 to 172 . The average number of colonies was 93 . The extreme variation between tube 6 and tube 7 was 72 per cent of the theoretical difference between the dilution in tubes 6 and 7 , but between tubes 5 and 6 the extreme variation was only 8 per cent.

\section{Viability of the leucocytes}

The presence and duration of life in the leucocytes under the conditions of the test were determined, roughly, by the use of unstained 
warm stage preparations. Blood to which pneumococci had been added was examined, as well as blood to which no pneumococci had been added, but which, in other respects, had been subjected to the same manipulations (tube 9). At the end of 6 hours rotation and 24 hours incubation the polynuclear leucocytes in the blood without pneumococci presented some changes. They did not appear as active as when first examined and the cytoplasm contained a moderate number of green refractive vacuoles. Observations on the same blood were made at intervals up to and including 72 hours. The polynuclear cells became more and more sluggish during that time; many lost the power of motility entirely, while others retained it to a slight degree. The

TABLE 2

Changes in the reading of the test after the end of the 24-hour period

\begin{tabular}{l|c|c|c}
\hline \multirow{2}{*}{$\begin{array}{c}\text { Amount of culture } \\
\text { suspension }\end{array}$} & \multicolumn{2}{|c|}{24 hours } & 72 hours \\
\cline { 2 - 4 } & Color change & Number of organisms & Color change \\
\hline 0.001 & + & $\infty$ & ++++ \\
0.0001 & $0^{*}$ & $\infty$ & ++++ \\
0.000075 & 0 & 4,500 & ++++ \\
0.00005 & 0 & 3,000 & ++++ \\
0.000025 & 0 & 7 & ++++ \\
0.00001 & 0 & 0 & 0 \\
\hline
\end{tabular}

${ }^{*} 0$, no change; + , slight change; ++ , darker red; +++ , deep red; ++++ , brown.

appearance of the lymphocytes was the same throughout, although they became motile toward the end of 72 hours incubation.

In the presence of actively growing pneumococci the changes mentioned above occurred much more quickly. The polynuclears were reduced in numbers and the remaining cells were filled with green vacuoles within six hours agitation and incubation. Practically all the polynuclears were destroyed within 18 to 24 hours incubation. Heist and his co-workers (2) made similar observations on the destruction of cells and fibrin by the pneumococci. They described it as apparent digestion by the pneumococci.

The less active the growth of the pneumococci the smaller the degree of destruction of the polynuclears. Where no growth occurred, that is, in blood which killed all the pneumococci which had been added to 
it, the polynuclears presented no changes in 24 hours in either motility or in the appearance of the cytoplasm, other than those changes observed in the control blood to which sterile gelatin-Locke's had been added.

The lymphocytes, as judged by the observation of warm-stage preparations and counts, were not affected by the growth of pneumococci in the blood. A number of unidentified cells developed in preparations with and without pneumococci.

\section{End point of the test}

Robertson and Sia made the final readings of their tests at the end of 72 hours, while other authors observed their results at the end of 18 or 24 hours. Several of our tests were read at both 24 and 48 hours, and a few were read at the end of 72 hours. The main difference in these readings is indicated in table 2. At the end of 72 hours there was no question about the color change which appeared in all tubes in which pneumococci remained viable. In 24 hours the color change caused by methemoglobin formation was not observed in some tubes in which many pneumococci were living and multiplying, but cultures at the end of 24 hours indicated all the tubes in which growth could continue.

For a time, blood broth cultures, blood agar plate cultures, mouse injection, and microscopic examination of the tube contents were used as indicators of the survival or death of the pneumococci. In regard to the first two, it was found that broth cultures were sometimes positive when plate cultures were negative and vice versa, but the difference was, at most, a matter of a very few organisms.

The results of mouse inoculations agreed with those of broth or plate cultures only when no serum protection for mice was present. The examination of stained smears could be used to indicate small numbers of organisms, as well as large numbers, if sufficient care was taken in the search.

The greater accuracy of blood agar plates, however, and the knowledge that the colonies indicated viable organisms seemed to us marked advantages.

Two controls are indicated in the diagram of the test table 1 as tubes 8 and 9 . Tube 8 contained plasma from heparinized blood 
seeded with the smallest number of pneumococci used in the test, namely, an average of 100 pairs. By this means any influence of the blood plasma upon the pneumococcus was found, such as agglutination, judged macroscopically. Tube 9 contained blood with gelatinLocke's solution, but no pneumococcus suspension. Growth in this control indicated that the patient's blood already contained organisms capable of proliferation. During approximately one-half of the work a third control, seeded with 100 pneumococci, was used, namely, red blood cells and plasma from which nearly all the white blood cells had been removed by centrifugalization. Growth in this control throughout our experiment was the same as in the plasma alone, and finally the use of this control was discontinued.

\section{Results of repeated tests on the same subjects}

A consideration of prime importance in the use of this test was a measure of its consistency when applied to the blood of one normal individual several different times. Two tests were made on the blood of five different individuals. In three of these comparisons agreement was perfect. In the two remaining comparisons there was a difference amounting to that between one tube and the next.

In further tests we had occasion to repeat the estimation of the pneumococcidal power of the blood of a laboratory worker who had no history of pneumococcus infection, but whose serum had some protection for the mouse. This test was done on 8 different days, with a total of 10 estimations. In only one instance was there any disagreement and that was to the same degree as above, namely, one tube. Since the method of making the culture dilution might account for this much variation, we felt that the results were satisfactory.

\section{Whole blood immunity in patients with no history of pneumonia}

Patients who gave no history of lobar pneumonia but who had been admitted for chronic or minor ailments were chosen for this part of the study. None of them had febrile infections at the time of the test. The diagnoses were dyspepsia, adhesions of the stomach, peptic ulcer, carcinoma, chorea, chronic cardiac valvular disease of rheumatic origin, chronic arthritis, chronic gonorrheal infection of the joints and urethra, acute nephritis, hypertension, arteriosclerosis, senility, neurasthenia, and urticaria. 
The number of pneumococci killed by $0.5 \mathrm{cc}$. of uncoagulated blood was determined for 27 different individuals. The results are given in table 3. The blood from 9 of the subjects killed no pneumococci; the blood from 17 killed from 100 organisms to 10,000 organisms, while the blood from one subject killed 100,000 organisms. Thus in hospital patients with no history of pneumonia, the pneumococcidal power of $0.5 \mathrm{cc}$. of whole blood for pneumococcus Type I varied from nil in one third of the subjects to 100 to 10,000 organisms in two thirds of the subjects, with one subject whose blood killed an even greater number.

TABLE 3

Pneumococcidal power of whole blood and protective power of serum in hospital patients with no history of pneumonia

\begin{tabular}{c|c|c|c|c|c|c}
\hline $\begin{array}{c}\text { Mouse protection in } \\
\text { serum x...D. }\end{array}$ & \multicolumn{5}{|c}{ Pneumococcidal power of whole blood (number of organisms killed) } \\
\cline { 2 - 7 } & 0 & 100 & $10^{3}$ & $10^{4}$ & $10^{5}$ & $10^{8}$ \\
\hline 0 & 3 & 1 & 5 & 4 & 0 & 0 \\
1 & 0 & 1 & 1 & 1 & 0 & 0 \\
10 & 0 & 0 & 1 & 1 & 0 & 0 \\
100 & 0 & 0 & 0 & 0 & 1 & 0 \\
$10^{3}$ & 0 & 0 & 0 & 0 & 0 & 0 \\
$10^{4}$ & 0 & 0 & 0 & 0 & 0 & 0 \\
Protection test not & 6 & 1 & 1 & 0 & 0 & 0 \\
performed & & & & & & \\
\hline Total........... & 9 & 3 & 8 & 6 & 1 & 0 \\
\hline
\end{tabular}

Reading from the left in the same table the results of the mouse protection tests are expressed as the number of minimal lethal doses (M.L.D.) of pneumococcus culture against which $0.2 \mathrm{cc}$. of the serum is effective in saving the life of a mouse. The serum for these tests was obtained from the subjects at the same time the whole blood sample was taken. The serum from 19 of the subjects was examined for protective power. Thirteen of the subjects gave no evidence of protection, while 6 had protective power against a small amount of culture. The measure of protection in normal individuals has been reported by Cecil and Austin (22) and by Clough (23, 24) with similar results.

The degree of pneumococcidal power can be compared to the degree 
of serum protection for the mouse in these 19 subjects. In the first place, while two thirds of the subjects had some whole blood pneumococcidal power, only one third had serum protection for mice.

To a certain extent, however, there is a relation between serum protection and whole blood immunity, inasmuch as among the six patients who had serum protection, all had some pneumococcidal power. The subject with the highest degree of protection (against 100 M.L.D.) also had the highest degree of whole blood pneumococcidal power $(100,000$ organisms). There is, however, a group of 13 subjects without any serum protection, 10 of whom had some whole blood pneumococcidal power. The degree of pneumococcidal power in the 10 subjects without serum protection ranged from 100 to 10,000 organisms, which includes the degrees found most frequently in the whole group of normal subjects.

These hospital patients with no history of lobar pneumonia were studied in lieu of strictly normal individuals to provide a base line with which to compare the degree of pneumococcidal power found in patients with pneumonia. We are encouraged to regard this base line as significant by the fact that this group of subjects presented measurements, in serum protection for mice, which were similar to measurements for groups of strictly normal individuals studied by other workers.

\section{SUMMARY}

1. A method is described for measuring the capacity of $0.5 \mathrm{cc}$. of uncoagulated human blood to kill pneumococci.

2. The blood of 17 out of 27 hospital patients who had not had lobar pneumonia killed from 100 to 10,000 virulent Type I pneumococci. The blood of 9 of the same group of patients killed no pneumococci.

3 . When both mouse protection and whole blood pneumococcidal power were measured, 6 subjects had both, 10 subjects had whole blood pneumococcidal power alone and 3 subjects had neither whole blood pneumococcidal power nor mouse protection.

\section{BIBLIOGRAPHY}

1. Wright, A. E. On Pharmo-Therapy and Preventive Inoculation Applied to Pneumonia in the African Native. New York, 1915, 78. 
2. Heist, G. D., Solis-Cohen, S. and Solis-Cohen, M., J. Immunol., 1918, iii, 261. The Bactericidal Action of Whole Blood with a New Technique for Its Determination.

3. Heist, G. D. and Solis-Cohen, S., J. Immunol., 1919, iv, 147. The Bactericidal Action of the Whole Blood of Rabbits Following Inoculations of Pneumococcus Bacterin.

4. Bull, C. G. and Tao, S. M., Am. J. Hyg., 1927, vii, 648. A Method for Determining the Antipneumococcal Properties of Whole Blood and the Protective Power of Immune Serum.

5. Wright, H. D., J. Path. and Bact., 1927, xxx, 185. Experimental Pneumococcal Septicemia and Antipneumococcal Immunity.

6. Goodner, K., J. Exp. Med., 1928, xlviii, 1. Experimental Intradermal Pneumococcus Infection in Rabbits.

7. Goodner, K., J. Exp. Med., 1928, xlviii, 413. Further Experiments with the Intradermal Pneumococcus Infection in Rabbits.

8. Blake, F. G. and Cecil, R. L., J. Exp. Med., 1920, xxxi, 403. Studies on Experimental Pneumonia. I. Production of Pneumococcus Lobar Pneumonia in Monkeys.

9. Blake, F. G. and Cecil, R. L., J. Exp. Med., 1920, xxxi, 499. Studies on Experimental Pneumonia. III. Spontaneous Pneumonia in Monkeys.

10. Cecil, R. L., and Blake, F. G., J. Exp. Med., 1920, xxxi, 519. Studies on Experimental Pneumonia. IV. Results of Prophylactic Vaccination Against Pneumococcus Pneumonia in Monkeys.

11. Cecil, R. L. and Blake, F. G., J. Exp. Med., 1920, xxxi, 657. Studies in Experimental Pneumonia. V. Active Immunity Against Experimental Pneumococcus Pneumonia in Monkeys Following Vaccination with Living Cultures of Pneumococcus.

12. Cecil, R. L. and Blake, F. G., J. Exp. Med., 1920, xxxi, 685. Studies on Experimental Pneumonia. VI. Active Immunity Following Experimental Pneumococcus Pneumonia in Monkeys.

13. Cecil, R. L. and Blake, F. G., J. Exp. Med., 1920, xxxii, 1. Studies on Experimental Pneumonia. VII. Treatment of Experimental Pneumococcus Type I Pneumonia in Monkeys with Type I Antipneumococcus Serum.

14. Robertson, O. H. and Sia, R. H. P., and Woo, S.T., J. Exp. Med., 1924, xxxix, 199. Studies on Pneumococcus Growth Inhibition. I. The Protective Action of Gelatin for Pneumococci in Suspension.

15. Robertson, O. H. and Sia, R. H. P., J. Exp. Med., 1924, xxxix, 219. Studies on Pneumococcus Growth Inhibition. II. A Method for Demonstrating the Growth Inhibitory and Bactericidal Action of Normal Serum-Leucocyte Mixtures.

16. Robertson, O. H., Woo, S. T. and Cheer, S. N., J. Exp. Med., 1924, xl, 487. Studies on Pneumococcus Growth Inhibition. IV. A Simplified Agitator for Growth Inhibition Tests with Serum-Leucocyte Mixtures; and Certain Modifications in the Technique of the Test. 
17. Howell, W. H. and Holt, E., Am. J. Physiol., 1918-1919, xlvii, 328. Two New Factors in Blood Coagulation; Heparin and Pro-Antithrombin.

18. Howell, W. H., Am. J. Physiol., 1924-1925, lxxi, 553. The Purification of Heparin and Its Presence in the Blood.

19. Ecker, E. E. and Gross, Paul, J. Infect. Dis., 1929, xliv, 250. Anti-complementary Power of Heparin.

20. Bull, C. G. and Bartual, L., J. Exp. Med. 1920, xxxi; 233. Pneumococcus Cultures in Whole Fresh Blood. I. The Retardative Effect of the Blood of Immune Animals and the Mechanism of the Phenomenon.

21. Gates, F. L., J. Exp. Med., 1920, xxxi, 105. A Method of Standardizing Bacterial Suspensions.

22. Cecil, R. L. and Austin, J. H., J. Exp. Med., 1918, xxviii, 19. Results of Prophylactic Inoculation Against Pneumococus in 12,519 Men.

23. Clough, P. W., J. Am. Med. Assoc., 1919, lxxiii, 785. Protective Power of Normal Human Serum Against Pneumococcus Infection.

24. Clough, P. W., Bull. Johns Hopkins Hosp., 1924, xxxv, 330. The Protective Power of Normal Human Serum Against Pneumococcus Infection. 